\title{
Risk Assessment in Young Hypertensives
}

\author{
Niteen V Deshpande
}

\begin{abstract}
Hypertension (HT) in young ( $<40$ years) is a significant problem in India. Preventing cardiovascular disease in these young hypertensives is a major challenge as management strategies for young hypertensives are not very clear. Risk assessment in young hypertensives is also limited as most of the risk assessment algorithms apply to population above 40 years. Unfortunately, we do not have a specific algorithm for Indian patients. The algorithm given by Joint British Societies (JBS-3) appears to be most suited for risk assessment in young Indian Hypertensive individuals. Additionally, multiple newer markers may be needed to understand the cardiovascular risk completely in the young hypertensive population.
\end{abstract}

Keywords: Cardiovascular risk assessment, Risk markers, Young hypertensives.

How to cite this article: Deshpande NV. Risk Assessment in Young Hypertensives. Hypertens J 2015;1(2):94-99.

Source of support: Nil

Conflict of interest: None

\section{INTRODUCTION}

Hypertension (HT) is the leading cause of cardiovascular $(\mathrm{CV})$ morbidity and mortality all over the globe. The incidence of HT is rising rapidly in the Asian countries including India. Approximately 30\% adults (> 20 years of age), urban as well as rural, are suffering from HT in India. ${ }^{1,2}$ A focused approach is required to manage this alarming increase in the incidence of hypertension and allied risk factors. Since the morbidity and mortality in HT results from CV events, every hypertensive must undergo assessment of risk for CV events. Aggressive management of high risk patients lowers the elevated risk. Aggressive management strategy today includes antihypertensive drugs, therapeutic lifestyle changes (TLC), statins and aspirin. Since TLC may be good for every adult, the issue of risk assessment boils down to use of statins and aspirin in young hypertensives. Thus,

\section{Director}

Cardiac Cath Lab, Spandan Heart Institute and Research Center, Nagpur, Maharashtra, India

Corresponding Author: Niteen V Deshpande, Director Cardiac Cath Lab, Spandan Heart Institute and Research Center, Dhantoli, Nagpur, Maharashtra, India, Phone: 07122443333, e-mail: nvdesh@hotmail.com the purpose of risk assessment is to identify individuals who will derive significant benefits from drug therapy.

\section{Young Hypertensive}

Although hypertension affects adult population $(>18$ years), most of the studies have included people over 50 years of age and limited data exists about younger people especially below 40 years of age. India is a young country with $65 \%$ of its population under 35 years of age. According to 2014 data, India has about 380 million individuals in the age group of 20 to 39 years. ${ }^{3}$ Assuming a modest estimate of hypertension prevalence of $20 \%$ in this population, number of hypertensive individuals in this age group exceeds 75 million. This large number of hypertensive individuals if not detected and treated in time will exert tremendous burden on the healthcare systems in the years to come. Unfortunately, data about risk assessment and management of hypertension in this age group is sparse. Detection of hypertension in this group of young individuals is a challenge as most of them are asymptomatic. Even when hypertension is detected, physicians may be reluctant to label them with a medical diagnosis that may have implications for the future insurance, jobs and perceptions of health. ${ }^{4}$ Besides, significance of isolated systolic hypertension (ISH) or isolated diastolic hypertension (IDH) at young age remains a matter of debate. However, recent data indicate that ISH even in young confers added CV risk. ${ }^{5}$ Long-term follow-up data from Chicago Heart Association Detection Project in Industry study ${ }^{6}$ showed that ISH in the young was associated with higher $\mathrm{CV}$ risk as compared to high normal blood pressure (BP) over follow-up of 31 years. Harvard Alumni Health study ${ }^{7}$ also showed elevated BP in the early life (university entry) was associated with higher risk of all-cause mortality over follow-up of more than 40 years. Similar observations have been reported from follow-up of more than 4 decades in Glasgow University students. ${ }^{8}$

\section{CARDIOVASCULAR RISK ASSESSMENT}

Management of hypertension today focuses more on management of global CV risk in addition to of control hypertension. ${ }^{9}$ Every hypertensive must be carefully evaluated for risk factor congregation and target organ damage at the baseline and during follow-up. Patient with target organ damage are high risk and need aggressive 
therapy. Young hypertensives without target organ damage (no e/o LVH, CKD or vascular disease) must also be evaluated at baseline and during follow-up for global $\mathrm{CV}$ risk. In the clinical practice, it is difficult to estimate contribution of individual risk factor to the global risk in a given patient. Risk assessment algorithms help to quantify risk of 10 years $\mathrm{CV}$ events by offering the risk in numerical form. Quantifying risk in absolute number makes it easy for the clinician to use appropriate risk management strategies. Of the numerous risk scores available, commonly used scores include Framingham Risk Score (FRS), ${ }^{10}$ European Systematic Coronary Risk Evaluation (SCORE), ${ }^{11}$ the 2013 ACC/AHA ASCVD risk score $^{12}$ and JBS-3 by Joint British Societies (Table 1). ${ }^{13,14}$ However, use of these risk score in young Indian population has certain limitations. Framingham score, ASCVD score and the SCORE tools are developed for population over 40 years of age and their applicability to people below 40 years is untested. The risk scores have been developed from large database like Framingham and have certain limitations in their applicability in the populations other than the original population. In this respect, the JBS-3 risk score developed by Joint British Societies can estimate risk for age group above 30 years up to 75 years. Further Indian ethnicity is specifically represented in JBS-3. A recent publication by Bansal et $\mathrm{al}^{15}$ have found that the JBS-3 score provides more accurate estimation of CV risk in Indian subjects than ASCVD score. Thus for calculation of global CV risk in young Indian hypertensive population JBS-3 appears to be the best suited model in absence of one developed in India. The risk calculator is readily available online and using this can be very handy in assessing risk in clinical practice. Calculated risk above $20 \%$ is indicative of significant risk to qualify the patient for drug therapy for primary prevention.

Table 1: Comparison of risk factors considered in different risk scores

\begin{tabular}{lllll}
\hline & FRS & ASCVD & JBS-3 & SCORE \\
\hline Age & $\checkmark$ & $\checkmark$ & $\checkmark$ & $\checkmark$ \\
Gender & $\checkmark$ & $\checkmark$ & $\checkmark$ & $\checkmark$ \\
Total cholesterol & $\checkmark$ & $\checkmark$ & $\checkmark$ & $\checkmark$ \\
HDL & $\checkmark$ & $\checkmark$ & $\checkmark$ & $\checkmark$ \\
Systolic BP & $\checkmark$ & $\checkmark$ & $\checkmark$ & $\checkmark$ \\
BP treatment & $\checkmark$ & $\checkmark$ & $\checkmark$ & $x$ \\
Diabetes & $\checkmark$ & $\checkmark$ & $x$ & $x$ \\
Smoking & $\checkmark$ & $\checkmark$ & $\checkmark$ & $\checkmark$ \\
Family h/o CVD & $x$ & $x$ & $\checkmark$ & $x$ \\
BMI & $x$ & $x$ & $\checkmark$ & $x$ \\
Region & $x$ & $x$ & $\checkmark$ & $\checkmark$ \\
Ethnicity & $x$ & $x$ & $\checkmark$ & $x$ \\
\hline
\end{tabular}

FRS: Framingham risk score ${ }^{10}$; SCORE: European systematic coronary risk evaluation ${ }^{11}$; ASCVD: The 2013 ACC/AHA ASCVD risk score ${ }^{12}$; JBS-3: Joint British Societies ${ }^{13}$
As the morbidity and mortality attached to hypertension results from $\mathrm{CV}$ events secondary to atherosclerosis, assessment of subclinical damage may give important clues in individuals with hypertension. Risk calculators, however, do not consider markers of subclinical atherosclerosis/arteriosclerosis like hs-CRP (exception-Reynolds Score), Aortic Stiffness measured by pulse wave velocity (PWV), ankle-brachial index, carotid intima-media thickness (cIMT) and microalbuminuria. Due to limited data on integration of these markers of vascular health in global risk prediction, it is unclear how a clinician should use this knowledge. European society of cardiology recommends that subjects with evidence of subclinical atherosclerosis should be allocated to higher risk category than that calculated with SCORE. ${ }^{16}$ An integrated approach to evaluation of CV risk is developed at Rasmussen Center for Cardiovascular disease prevention at the University of Minnesota, $\mathrm{MN}^{17}$ which involves 10 tests (Table 2) to generate a disease score (DS). Each test is scored as point 0 for normal, 1 for borderline abnormal and 2 for abnormal result. A disease score above 6 is considered high risk score and is related to higher occurrence of morbid CV events over 6 years follow-up.

Considerable data are accumulated about role of these added risk markers over past 2 decades. Estimation of these additional markers in young hypertensives can help in evaluating $\mathrm{CV}$ risk further especially for patients in intermediate risk category. In absence of large randomized data on addition of these markers to the classic risk scores, a clinician must use his clinical judgment in interpreting results of these tests on case to case basis. Current status of these markers is discussed briefly in the following section.

\section{Abdominal Obesity}

Abdominal obesity is now recognized as an important contributor in development of diabetes and atherosclerosis especially in Asian Indians. Traditionally, obesity is

Table 2: An integrated approach for $C V$ risk assessment developed by Rasmussen Center, University of Minnesota
1. Resting sitting blood pressure
2. Small artery elasticity (pulse wave analysis)
3. Large artery elasticity (pulse wave analysis)
4. Exercise BP response (three minutes, 5 METS)
5. Carotid intima-media thickness + plaques
6. Retinal digital photograph
7. Urine sample for albumin/creatinine ratio
8. Electrocardiogram
9. Left ventricular ultrasound for thickness, mass
10. Blood sample for N-terminal pro b-type natriuretic peptide 
defined on the basis of body mass index (BMI). However, recent findings indicate that waist circumference and/or waist to hip ratio may provide a better estimate of abdominal obesity and risk of cardiovascular disease. ${ }^{18}$ Estimation of BMI alone may underestimate risk in South Asians as they tend to have increased visceral fat and greater insulin resistance at similar levels of BMI as compared to Europeans. ${ }^{19}$ In fact, insulin resistance is commonly noted in south Asians at BMI levels below $25 \mathrm{~kg} / \mathrm{m}^{2} .^{20}$ Population specific definitions of abdominal obesity have been incorporated into diagnostic criteria for metabolic syndrome by the National Cholesterol Education Program Adult Treatment Panel III in the United States and the cut off for waist circumference for Asian men and women is 90 and $80 \mathrm{~cm} .{ }^{21}$ Simple inexpensive measurement of waist circumference in the young hypertensive patients can be used as a target thus helping in advising about weight reduction through regular exercise and diet modification.

\section{Aortic Stiffness and Pulse Wave Velocity}

Aortic stiffness is a marker of arterial stiffening (arteriosclerosis) and indicates arterial wall damage in the subclinical stage. ${ }^{22}$ Measurement of carotid femoral pulse wave velocity (CFPWV) reflects stiffness of large arteries and is a strong predictor of future $\mathrm{CV}$ events. ${ }^{23}$ Carotid femoral pulse wave velocity above $10 \mathrm{~m} / \mathrm{s}$ is now considered abnormal according to the expert consensus statement of ESC (2012). Significant data indicate that use of CFPWV improves risk prediction in patients with hypertension without overt cardiovascular disease. ${ }^{23-25}$ With measurement of CFPWV in the Framingham study, ${ }^{26} 15.7 \%$ of patients at intermediate risk could be reclassified into higher $(14.3 \%)$ or lower $(1.4 \%)$ risk. In another meta-analysis, ${ }^{27} 19$ and $22 \%$ of the intermediate risk individuals were reclassified into higher or lower quartiles of risk. However, data on modulation of aortic stiffness and its impact on CV risk is limited ${ }^{28}$ and will be evaluated in 4 years prospective Strategie de prevention Cardiovasculaire Basėe sur la Rigidité Arterielle (SPARTE) Study. Smaller studies indicate that ACE inhibitors, angiotensin receptor blockers and spironolactone reduce the aortic stiffness beyond their BP reducing effects while beta-blockers limit de-stiffening of arterial wall and in fact can increase it in some patient population. ${ }^{22}$

\section{C-reactive Protein}

High sensitivity C-reactive protein (hs-CRP) is an inflammatory biomarker which independently predicts future vascular events in diverse population ranging from healthy individuals ${ }^{29,30}$ to patients with acute coronary syndromes regardless of LDL cholesterol levels. Utility of elevated levels of hs-CRP over and above traditional risk factors in predicting 10 year CV events was clearly demonstrated in CV health study ${ }^{31}$ which included men and women above 65 years of age without vascular disease. Data from JUPITER trial ${ }^{32}$ shows that treatment of apparently healthy individuals above 50 years of age and elevated levels of hs-CRP ( $>2 \mathrm{mg} / \mathrm{l})$ with rosuvastatin reduces major $\mathrm{CV}$ events significantly. Although utility of hs-CRP in younger individuals is not specifically demonstrated in a large randomized study, it should be equally powerful marker in this population too. The 2013 ACC/AHA guidelines ${ }^{12}$ on the assessment of $\mathrm{CV}$ risk do not recommend use of this marker routinely in low risk individuals. However, it remains a very useful marker in people with intermediate risk (FRS 5 to $20 \%$ ) to identify candidates for statin therapy (ACC/AHA IIb/B).

\section{Carotid Intima-Media Thickness}

Imaging the carotid arteries provide a window to detect subclinical atherosclerosis by direct visualization of wall thickening measured as cIMT. ${ }^{33}$ Elevated cIMT and/or presence of plaques in carotid arteries has been shown to predict occurrence of stroke and myocardial infarction independent of traditional risk factors. ${ }^{34-37}$ The relationship between cIMT and CV events is continuous and determining a threshold for $\mathrm{CV}$ events is rather arbitrary. Although, ESC guidelines 2007 considered cIMT $>0.9 \mathrm{~mm}$ indicative of existing abnormality, threshold value for high $\mathrm{CV}$ risk was $>1.0 \mathrm{~mm}$ in the middle aged patients of European Lacidipine Atherosclerosis (ELSA) study. ${ }^{38}$ In the atherosclerosis in the communities (ARIC) study $^{39}$ addition of cIMT and carotid plaques added little value for predicting $\mathrm{CV}$ events and reclassifying the patients into another risk category. Despite of these uncertainties a recent systematic review indicates added predictive value of cIMT in asymptomatic individuals at intermediate risk. ${ }^{40}$

\section{Ankle-brachial Index (ABI)}

Ratio of systolic BP measured at the ankle to brachia systolic BP provides useful assessment of peripheral vasculature. Ankle-brachial index $(\mathrm{ABI})<0.9$ indicates advanced peripheral vascular disease ${ }^{41}$ and is related to 2-fold increase in the 10 years $\mathrm{CV}$ risk in each Framingham category. ${ }^{42}$ Despite this, addition of ABI to Framingham risk score improved risk prediction only marginally. Thus, measurement of ABI although indicates peripheral atherosclerosis, integration of this information in risk assessment has limited value in nonselected patients. A low ABI may help patients in the intermediate risk 
Risk Assessment in Young Hypertensives

category to be reclassified into higher risk category and eligible for aggressive treatment.

\section{MICROALBUMINURIA}

Microalbuminuria is a marker for generalized vascular dysfunction. It is also an important, independent marker for endothelial dysfunction and CV diseases. ${ }^{43}$ Traditionally, it is used in diabetic patients to monitor the development and progression of kidney disease. An international survey i-SEARCH${ }^{44}$ conducted to evaluate microalbuminuria in approximately 22000 hypertensive patients with or without CV disease showed the prevalence of microalbuminuria ranging from 53 to $71 \%$ patients. Highest rate of microalbuminuria was observed in patients with uncontrolled hypertension. Microalbuminuria had been shown to correlate with 4-fold increased risk of ischemic heart disease among hypertensive or borderline hypertensive subjects in 10 years follow-up analysis of all subjects with untreated arterial hypertension or borderline hypertension identified within the World Health Organization (WHO) multinational monitoring of trends and determinants in cardiovascular disease (MONICA) study. ${ }^{43}$ Microalbuminuria is typically defined as a 24 hours urinary albumin excretion rate of 30 to $300 \mathrm{mg}(20-200 \mu \mathrm{g} /$ min) or urinary albumin creatinine ratio (UACR) of 2.5 to $30 \mathrm{mg} / \mathrm{mmol}$ in men 3.5 to $30 \mathrm{mg} / \mathrm{mmol}$ in women. The assessment of UACR along with the other markers of subclinical atherosclerosis can be helpful in global risk assessment of hypertensive patients. Urinary albumin excretion should be measured regularly in a hypertension clinic, and a rigorous control of BP and of other atherosclerotic risk factors is recommended in hypertensive patients with microalbuminuria. Reduction in microalbuminuria with ACE/ARB inhibitors have been shown to reduce the progression of atherosclerosis. ${ }^{45,46}$

Large population of young hypertensive patients in India demands early detection and optimal management to reduce subsequent morbidity and mortality. Unfortunately, strategies to optimally manage these young patients are lacking and we need to extrapolate the existing data about managing $\mathrm{CV}$ risk derived from older patient population. On one hand overestimation of $\mathrm{CV}$ risk in a young hypertensive can unnecessarily expose him to long-term drug therapy, on the other hand inadequate evaluation may lead to loss of opportunities of preventing CV events. Ideally, we need to develop specific strategies for this large pool of young hypertensive patients by systematic follow-up. Till such data are available, one can use the existing risk calculators (like JBS-3) as an initial tool to identify high risk group
Flow Chart 1: Suggested algorithm for risk assessment in young hypertensives

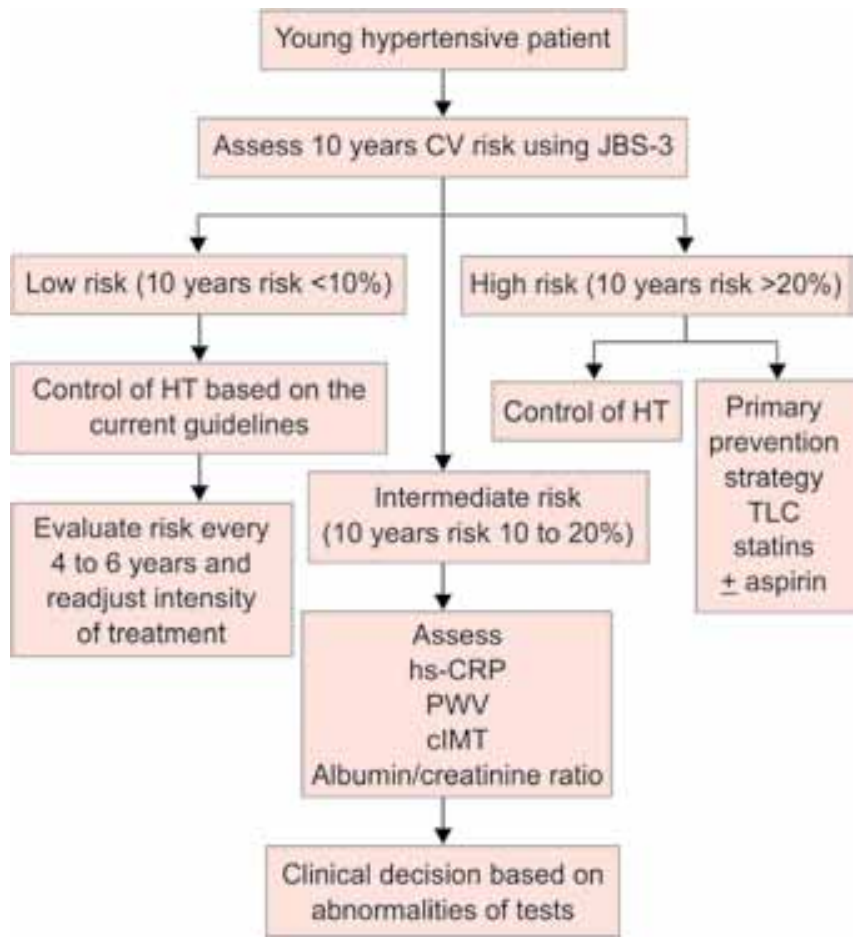

who clearly will benefit from intensive TLC and drug therapy (Flow Chart 1). Individuals in the intermediate risk category can undergo further evaluation for subclinical atherosclerosis. Intermediate risk patients exhibiting sub-clinical atherosclerosis are likely to benefit by aggressive preventive strategy including drugs like statins. Low risk patients may be followed up on TLC and antihypertensive therapy for adequate control of hypertensive with periodic assessment of risk development every 4 to 6 years.

\section{REFERENCES}

1. Anchala R, Kannuri NK, et al. Hypertension in India: a systematic review and meat-analysis of prevalence, awareness and control of hypertension. J Hypertension 2014;32:1170-1177.

2. Gupta R. Trends in hypertension epidemiology in India. J Human Hypertension 2004;18:73-78.

3. Available at: http://unstats.un.org/unsd/demographic/ products/dyb/dyb2.htm.

4. Bibbins-Domingo K, Pletcher MJ. Blood pressure matters, even during young adulthood. J Am Coll Cardiol 2011;58: 2404-2405.

5. Weber MA. Interpreting blood pressure in young adults. J Am Coll Cardiol 2015;65:336-338.

6. Yano Y, Stamler J, et al. Isolated systolic hypertension in young and middle aged adults and 31 years risk for cardiovascular mortality. The Chicago Heart Association Detection Project in Industry Study. J Am Coll Cardiol 2015;65:327-335.

7. Gray L, Lee I-min, Sesso HD, Batty GD. Blood pressure in early adulthood, hypertension in middle age and future 
cardiovascular disease mortality Harvard Alumni Health Study (HAHS). J Am Coll Cardiol 2011;58:2396-2403.

8. McCarron P, Smith GD, Okasha M, McEwen J. Blood pressure in young adulthood and mortality from cardiovascular disease. Res Letter Lancet 2000;355:1430-1431.

9. Lloyd-Jones DM. Cardiovascular risk prediction: basic concepts, current status, and future directions. Circulation 2010;121:1768-1777.

10. D'Agostino RB Sr, Vasan RS, Pencina MJ, et al. General cardiovascular risk profile for use in primary care: the Framingham Heart Study. Circulation 2008;117:743-753.

11. Conroy PM, Pyorala, Fitzgerald AP, et al. Estimation of 10 years risk of fatal cardiovascular disease in Europe: the SCORE project. Eur Heart J 2003;24:987-1003.

12. Goff DC, Lloyd-Jones DM, et al. 2013 ACC/AHA guideline on the assessment of cardiovascular risk: a report of the American College of Cardiology/American Heart Association Task Force on practice guidelines. Circulation 2014;129 (suppl 2):S49-S72

13. JBS3 Board. Joint BritishSocieties' consensus recommendations for the prevention of cardiovascular disease (JBS3). Heart 2014;100:ii1-ii67.

14. Hippisley-Cox J, Coupland C, et al. Predicting cardiovascular risk in England and Wales: prospective derivation and validation of QRISK2. BMJ 2008;336:a332.

15. Bansal M, Kasliwal RR, Trehan N. Relationship between different cardiovascular risk score and measures of subclinical atherosclerosis in an Indian population. Ind Heart J 2015;67:332-340.

16. Sehestedt $\mathrm{T}$, Jeppesen J, et al. Risk prediction is improved by adding markers of subclinical organ damage to SCORE. Eur Heart J 2010;31:883-891.

17. Cohn JN. Prevention of cardiovascular disease. Trends Cardiovasc Med 2015;25:436-442.

18. Yusuf S, Hawken S, et al. INTERHEART study investigators. obesity and risk of myocardial infarction in 27000 participants from 52 countries: a case control study. Lancet 2005;366: 1640-1649.

19. McKeigue PM, Shah B, et al. Relation of central obesity and insulin resistance with high diabetes prevalence and cardiovascular risk in south Asians. Lancet 1991;337:382-386.

20. Snehalata C, Vishwanatahan V, Ramachandran A. Cutoff values normal anthropometric variables in Asian Indian adults. Diabetes Care 2003;26:1380-1384.

21. The IDF consensus worldwide definition of the metabolic syndrome. International Diabetes Federation 2005. Available at: http://www.idf.org/home.

22. Laurent $S$, Briet $M$, Boutouyrie P. Arterial stiffness as surrogate end point-needed clinical trials. Hypertens 2012;60: 518-522.

23. Vlachopoulos C, Aznaouridis K, et al. Prediction of cardiovascular events and all-cause mortality with arterial stiffness: a systematic review and meta-analysis. J Am Coll Cardiol 2010;55:1318-1327.

24. Mattece-Raso FU, van der Cammen TJ, et al. Arterial stiffness and risk of coronary heart disease and stroke: the Rotterdam heart study. Circulation 2006;113:657-663.

25. Boutouyrie P, Tropeano AI, et al. Aortic stiffness is an independent predictor of primary coronary events in hypertensive patients-a longitudinal study. Hypertens 2002;39:10-15.

26. Mitchell GF, Hwang SJ, et al. Arterial stiffness and cardiovascular events-the Framingham heart study. Circulation 2010;121:505-511.

27. Ben Schlomo Y, Spears M, et al. Predicative value of pulse wave velocity for cardiovascular events in 15520 subjects: an individual participant meta-analysis on behalf of the PWV collaborative group. J Hypertens 2010;28(Suppl A):E466.

28. Ait-Oufella $\mathrm{H}$, Collon $\mathrm{C}$, et al. Long-term reduction in aortic stiffness: a 5.3 years follow-up in routine clinical practice. J Hypertens 2010;28:2336-2341.

29. Ridker PM. High sensitivity C-reactive protein: potential adjunct for global risk assessment in the primary prevention of cardiovascular disease. Circulation 2001;103:1813-1818.

30. Ridker PM, Rifai N, et al. Comparison of c-reactive protein and low-density lipoprotein cholesterol levels in the prediction of first cardiovascular events. N Eng J Med 2002;347:1557-1565.

31. Cushman M, Arnold Am, et al. C-reactive protein and 10-year incidence of coronary heart disease in older men and women: the cardiovascular health study. Circulation 2005;112:25-31.

32. Ridker PM, Danielson E, et al. Rosuvastatin to prevent vascular events in men and women with elevated C-reactive protein. N Eng J Med 2008;359:2195-2207.

33. $2013 \mathrm{ESH} / \mathrm{ESC}$ guidelines for the management of arterial hypertension. The task force for the management of arterial hypertension of the European Society of Hypertension (ESH) and of the European Society of Cardiology (ESC). J Hypertens 2013;31:1281-1357.

34. Bots MI, Hoes AW, et al. Common carotid intima media thickness and risk of stroke and myocardial infarction: the Rotterdam study. Circulation 1997:96:1432-1437.

35. Sehestedt $\mathrm{T}$, Jeppesen J, et al. Risk prediction is improved by adding markers of sub clinical organ damage to SCORE. Eur Heart J 2010;31:883-891.

36. O'Leary DH, Polak JF, et al. Carotid artery intima and media thickness as a risk factor for myocardial infarction and stroke in older adults. Cardiovascular health study collaborative research group. N Eng J Med 199;340:14-22.

37. Sesso HD, Stampfer MJ, et al. Systolic and diastolic blood pressure, pulse pressure and mean arterial pressure as predictors of cardiovascular disease risk in men. Hypertension 2000;36:801-807.

38. Zanchetti A, Bond MG, et al. Calcium antagonist lacidipine slows down progression of asymptomatic carotid atherosclerosis. Principal results of the European lacidipine study of atherosclerosis (ELSA) a randomized double blind long-term trial. Circulation 2002;106:2422-2427.

39. Nambi V, Chambless L, et al. Carotid intima-media thickness and presence or absence of plaque improves prediction of coronary heart disease risk: the atherosclerosis risk in communities (ARIC) study. J Am Coll Cardiol 2010;55:1600-1607.

40. Peters SA, den Ruijter HM, et al. Improvements in risk stratification for the occurrence of cardiovascular disease by imaging subclinical atherosclerosis: a systematic review. Heart 2012;98:177-184.

41. Feringa $\mathrm{HH}, \mathrm{Bax} J J$, et al. The long-term prognostic value of the resting and post-exercise ankle brachial index. Arch Intern Med 2006;166:529-535. 
42. Fowkes FG, Murray GD, et al. Ankle brachial index combined with Framingham risk score to predict cardiovascular events and mortality: a meta-analysis. JAMA 2008;300: 197-208.

43. Jensen JS, Feldt-Rasmussen B, et al. Arterial hypertension, microalbuminuria and risk of ischemic heart disease. Hypertens 2000;35:898-903.

44. Bohm M, Thoenes M, et al. Association of cardiovascular risk factors with microalbuminuria in hypertensive individuals: the i-SEARCH global study. J Hypertens 2007;25: 2317-2324.

45. Ibsen $\mathrm{H}$, Olsen $\mathrm{MH}$, et al. Reduction in albuminuria translets to reduction in cardiovascular events in hypertensive patients. Losartan intervention for endpoint reduction in hypertension study. Hypertens 2005;45:198-202.

46. Leoncini G, Martinoli C, et al. Changes in renal resistive index and urinary albumin excretion in hypertensive patients under long-term treatment with lisinopril or Nifedipine GITS. Nephron 2002;90:169-173. 